\title{
Should you advocate for hepatocellular carcinoma surveillance in patients with alcohol-related liver disease or non-alcoholic fatty liver disease?
}

\author{
Ju Hyun Shim \\ Department of Gastroenterology, Asan Liver Center, Asan Medical Center, University of Ulsan College of Medicine, Seoul, Korea
}

Keywords: Hepatocellular carcinoma; Non-alcoholic fatty liver disease; Non-alcoholic steatohepatitis; Alcoholic liver disease; Surveillance

\section{See Article on Page 196}

Although hepatocellular carcinoma (HCC) is mainly caused by hepatitis B or C virus infection, the incidence of HCC related to alcohol abuse or non-alcoholic fatty liver disease (NAFLD) has risen markedly in recent decades. The Global Burden of Disease Study 2015 reported that $30 \%$ of new primary liver cancer cases were attributable to alcohol.' In Korea, $2.4-10.9 \%$ of HCC cases were attributed to alcohol use. ${ }^{2}$ In addition, a year-over-year increase in the proportion of HCC cases attributable to NAFLD has been documented in several epidemiological studies. ${ }^{3,4}$ It is likely that the relative contribution of alcohol or NAFLD to primary liver cancer will increase worldwide, due to the improved efficacy of anti-hepatitis $B$ and $C$ treatments.

Regarding this issue, Kumar et al. ${ }^{5}$ compared the demographic and tumor characteristics, receipt of surveillance program, and survival outcomes between patients with HCC related to non-alcoholic steatohepatitis (NASH) and alcoholic liver disease (ALD) who were selected from the prospective HCC database of Singapore General Hospital. Patients with NASH-related HCC were older, and had higher prevalence of features suggestive of metabolic syndromes such as diabetes and hyperlipidemia. Notably, the majority of cases caused by NASH or ALD were associated with deficient surveillance as well as HCC detected late after the development of symptoms. These delays resulted in advanced-stage tumors at diagnosis, which were not amenable to potentially curative therapy and predicted poor outcomes.

Although this study offers additional insight into the characteristics of HCC in patients with NASH or ALD, it still had some bias and limitations. First, there was no data to compare NASH or ALD-related HCC and those caused by more common etiologies, including hepatitis B and hepatitis C. Second, the criteria for NASH diagnosis used in this study were somewhat ambiguous. NASH is standardly defined as the presence of $5 \%$ hepatic steatosis and inflammation with hepatocyte injury (e.g., ballooning), with or without fibrosis. ${ }^{6}$ Although liver biopsy is an essential element in the diagnosis of $\mathrm{NASH}_{1}^{7,8}$ this study did not mention any

\footnotetext{
Abbreviations:

ALD, alcoholic liver disease; HCC, hepatocellular carcinoma; NAFLD, nonalcoholic fatty liver disease; NASH, non-alcoholic steatohepatitis
}

\section{Corresponding author : Ju Hyun Shim}

Department of Gastroenterology, Asan Liver Center, Asan Medical Center, University of Ulsan College of Medicine, 88 Olympic-ro 43-gil, Songpagu, Seoul 05505, Korea

Tel: +82-2-3010-3190, Fax: +82-2-485-5782

E-mail:s5854@amc.seoul.kr

https://orcid.org/0000-0002-7336-1371 
histological examination of surrounding liver tissues. These concerns should be considered when interpreting the results of this study.

Currently, limited data are available to suggest an optimal guidance for surveilling patients with NAFLD/NASH or ALD for HCC. Indeed, less than one-fifth of the 99 patients included in this study were surveilled or screened for HCC. A recently updated American Gastroenterology Association clinical practice guideline recommends that HCC screening should be considered in all NAFLD patients with cirrhosis as well as advanced liver fibrosis, where non-invasive approaches combining serological tests with elastography examinations in staging liver fibrosis in NAFLD are preferred. ${ }^{9}$ On the other hand, a European multicenter study showed that 1- and 2-year cumulative incidence of HCC development in patients with alcoholic cirrhosis were up to $1.8 \%$ and $5.2 \%$, respectively, which were high enough to justify surveillance for the disease in these patients. ${ }^{10}$ On the contrary, a need for regular surveillance remains questionable in non-cirrhotic patients with NASH or ALD. In this study, more than $10 \%$ of HCC patients had no feature of cirrhosis, and several previous studies also found that HCC may develop in NAFLD/NASH patients without cirrhotic background. " However, the implementation of HCC surveillance is not formally accepted in non-cirrhotic patients with chronic liver disorders of any etiology, due to its low cost-effectiveness. ${ }^{12,13}$

Future studies should address the factors on which to base an optimal frequency and intensity of surveillance for patients with advanced fibrosis or cirrhosis attributed to hepatic fat or alcohol.

\section{Conflicts of Interest}

The author has no conflicts to disclose.

\section{REFERENCES}

1. Global Burden of Disease Liver Cancer Collaboration, Akinyemiju T, Abera S, Ahmed M, Alam N, Alemayohu MA, et al. the burden of primary liver cancer and underlying etiologies from 1990 to 2015 at the global, regional, and national level: results from the global burden of disease study 2015. JAMA Oncol 2017;3:1683-1691.
2. Kim BH, Park JW. Epidemiology of liver cancer in South Korea. Clin Mol Hepatol 2018;24:1-9.

3. Dyson J, Jaques B, Chattopadyhay D, Lochan R, Graham J, Das D, et al. Hepatocellular cancer: the impact of obesity, type 2 diabetes and a multidisciplinary team. J Hepatol 2014;60:110-117.

4. Petrick JL, Braunlin M, Laversanne M, Valery PC, Bray F, McGlynn $K A$. International trends in liver cancer incidence, overall and by histologic subtype, 1978-2007. Int J Cancer 2016;139:1534-1545.

5. Kumar R, Goh BG, Kam JW, Chang PE, Tan CK. Comparisons between non-alcoholic steatohepatitis and alcohol-related hepatocellular carcinoma. Clin Mol Hepatol 2020;26:196-208.

6. Chalasani N, Younossi Z, Lavine JE, Charlton M, Cusi K, Rinella M, et al. The diagnosis and management of nonalcoholic fatty liver disease: practice guidance from the American Association for the Study of Liver Diseases. Hepatology 2018;67:328-357.

7. European Association for the Study of the Liver (EASL); European Association for the Study of Diabetes (EASD); European Association for the Study of Obesity (EASO). EASL-EASD-EASO clinical practice guidelines for the management of non-alcoholic fatty liver disease. J Hepatol 2016;64:1388-1402.

8. Korean Association for the Study of the Liver (KASL). KASL clinical practice guidelines: management of nonalcoholic fatty liver disease. Clin Mol Hepatol 2013;19:325-348.

9. Loomba R, Lim JK, Patton H, El-Serag HB. AGA clinical practice update on screening and surveillance for hepatocellular carcinoma in patients with nonalcoholic fatty liver disease: expert review. Gastroenterology 2020 Jan 29. [Epub ahead of print]

10. Ganne-Carrié N, Chaffaut C, Bourcier V, Archambeaud I, Perarnau $\mathrm{JM}$, Oberti $F$, et al. Estimate of hepatocellular carcinoma incidence in patients with alcoholic cirrhosis. J Hepatol 2018;69:1274-1283.

11. Stine JG, Wentworth BJ, Zimmet A, Rinella ME, Loomba R, Caldwell $\mathrm{SH}$, et al. Systematic review with meta-analysis: risk of hepatocellular carcinoma in non-alcoholic steatohepatitis without cirrhosis compared to other liver diseases. Aliment Pharmacol Ther 2018;48:696703.

12. Kanwal F, Kramer JR, Mapakshi S, Natarajan Y, Chayanupatkul M, Richardson PA, et al. Risk of hepatocellular cancer in patients with non-alcoholic fatty liver disease. Gastroenterology 2018;155:1828 1837.e2.

13. Reig M, Gambato M, Man NK, Roberts JP, Victor D, Orci LA, et al. Should patients with NAFLD/NASH be surveyed for HCC? Transplantation 2019;103:39-44. 